\title{
High-energy neutrinos from multibody decaying dark matter
}

\author{
Nagisa Hiroshima, ${ }^{1,2}$ Ryuichiro Kitano, ${ }^{2,3}$ Kazunori Kohri, ${ }^{2,3,4}$ and Kohta Murase ${ }^{5,6}$ \\ ${ }^{1}$ Institute for Cosmic Ray Research, The University of Tokyo, \\ 5-1-5 Kashiwanoha, Kashiwa, Chiba 277-8582, Japan \\ ${ }^{2}$ Institute of Particle and Nuclear Studies, Theory Center, \\ KEK, 1-1 Oho, Tsukuba, Ibaraki 305-0801, Japan \\ ${ }^{3}$ The Graduate University for Advanced Studies (Sokendai), 1-1 Oho, Tsukuba 305-0801, Japan \\ ${ }^{4}$ Rudolf Peierls Centre for Theoretical Physics, The University of Oxford, \\ 1 Keble Road, Oxford OX1 3NP, United Kingdom \\ ${ }^{5}$ Department of Physics; Department of Astronomy and Astrophysics; \\ Center for Particle and Gravitational Astrophysics, The Pennsylvania State University, \\ University Park, Pennsylvania 16802, USA \\ ${ }^{6}$ Yukawa Institute for Theoretical Physics, Kyoto University, Kyoto 606-8502, Japan
}

(Received 27 September 2017; published 10 January 2018)

\begin{abstract}
Since the report of the $\mathrm{PeV}-\mathrm{TeV}$ neutrinos by the IceCube Collaboration, various particle physics models have been proposed to explain the neutrino spectrum by dark matter particles decaying into neutrinos and other standard model particles. In such scenarios, simultaneous $\gamma$-ray emission is commonly expected. Therefore, multimessenger connections are generally important for the indirect searches of dark matters. The recent development of $\gamma$-ray astronomy puts stringent constraints on the properties of dark matter, especially by observations with the Fermi $\gamma$-ray satellite in the last several years. Motivated by the lack of $\gamma$-ray as well as the shape of the neutrino spectrum observed by IceCube, we discuss a scenario in which the $\mathrm{DM}$ is a PeV scale particle which couples strongly to other invisible particles and its decay products do not contain a charged particle. As an example to realize such possibilities, we consider a model of fermionic dark matter that decays into a neutrino and many invisible fermions. The dark matter decay is secluded in the sense that the emitted products are mostly neutrinos and dark fermions. One remarkable feature of this model is the resulting broadband neutrino spectra around the energy scale of the dark matter. We apply this model to multi-PeV dark matter, and discuss possible observable consequences in light of the IceCube data. In particular, this model could account for the large flux at medium energies of $\sim 10-100 \mathrm{TeV}$, possibly as well as the second peak at PeV, without violating the stringent $\gamma$-ray constraints from Fermi and air-shower experiments such as CASA-MIA.
\end{abstract}

DOI: $10.1103 /$ PhysRevD.97.023006

\section{INTRODUCTION}

High-energy neutrinos are powerful probes of the Universe in astrophysics and cosmology. They enable us to study physical processes in their sources because they interact with other particles only through the weak interaction. Searches for astrophysical and cosmogenic highenergy neutrinos have been drastically advanced in this decade. The full IceCube detector was completed in 2010. Since the first announcement of the detection of highenergy cosmic neutrinos [1,2], its evidence has been accumulated [3-7]. It is worth noting that some $\mathrm{PeV}$

Published by the American Physical Society under the terms of the Creative Commons Attribution 4.0 International license. Further distribution of this work must maintain attribution to the author(s) and the published article's title, journal citation, and DOI. Funded by SCOAP . neutrino events have been detected [3,7]. These neutrinos can provide us with not only information about the astrophysical accelerators which have abilities to generate cosmic rays with $\mathcal{O}(10-100) \mathrm{PeV}$, but also some hints about physics beyond the standard model.

There are numbers of interpretations on the origin of the $\mathrm{TeV}-\mathrm{PeV}$ neutrinos (see a review [8]). So far no clustering in time and/or space has been seen. The observed diffuse neutrino intensity above $\sim 0.1 \mathrm{PeV}$ is $E_{\nu}^{2} \Phi_{\nu} \sim$ $3 \times 10^{-8} \mathrm{GeV} \mathrm{cm}^{-2} \mathrm{~s}^{-1} \mathrm{sr}^{-1}$ for the sum of three flavors, and the spectrum can be approximately described by a single power law with its spectral index, $s_{\nu} \simeq 2.0-2.7$ up to $\mathcal{O}(1) \mathrm{PeV}[4,6,7,9]$. Remarkably, the latest analysis focusing on medium-energy starting events below $0.1 \mathrm{PeV}$ $[4,5,10-12]$ as well as the conventional shower analysis $[5,9]$ have indicated that the neutrino flux in the $30 \mathrm{TeV}$ range is as high as $E_{\nu}^{2} \Phi_{\nu} \sim 10^{-7} \mathrm{GeV} \mathrm{cm}^{-2} \mathrm{~s}^{-1} \mathrm{sr}^{-1}$. Consequently, for a power-law fitting, the medium-energy 
spectral index below $\sim 0.1 \mathrm{PeV}$ is $s_{\mathrm{ME}} \sim 2.5-2.7$, which suffers from a $\sim 3 \sigma$ tension with the high-energy spectral index, $s_{\mathrm{HE}} \sim 2.0-2.2$ [7]. The flavor ratio is consistent with $\nu_{e}: \nu_{\mu}: \nu_{\tau} \approx 1: 1: 1[5,13]$, as expected in the long baseline limit of neutrino oscillation.

In astrophysical models, those high-energy neutrinos can be produced by proton-proton $(p p)$ and/or proton-gamma $(p \gamma)$ interactions [14-16] induced by accelerated primary cosmic rays. In this kind of scenarios, extragalactic sources may be favored because the observed neutrinos are isotropically distributed. In particular, cosmic-ray reservoirs such as starburst galaxies and galaxy clusters give a grandunified picture of the observed neutrinos, $\gamma$ rays, and ultra-high energy cosmic rays $[14,17,18]$. On the other hand, important constraints on astrophysical models for IceCube's neutrinos have been placed by the diffuse isotropic $\gamma$-ray background (IGRB), which is measured by the Fermi $\gamma$-ray satellite [19]. In either $p p$ or $p \gamma$ process, $\gamma$ rays should be commonly generated and their fluxes are known to trace the neutrino flux approximately in a model-independent way. This fact inevitably induces a severe problem that those $\gamma$-rays may exceed the measured IGRB flux at around 10-100 GeV [14]. This is especially the case if the neutrino flux excess at medium energies is real, and hidden cosmic-ray accelerators, which are opaque to $\gamma$ rays, are needed to reconcile the neutrino and $\gamma$-ray data [16].

Not only astrophysical interpretations but also the new physics models are feasible at present. In particular, high-energy neutrinos can be emitted through decays of a long-lived heavy particle with a mass of $\mathcal{O}(1)-\mathcal{O}(10) \mathrm{PeV}$ [20-39], of which the dark matter (DM) is expected to consist. Using the IceCube data, lower bounds on the lifetimes of $\mathrm{PeV}$ or heavier DMs have been obtained, $\tau \gtrsim 10^{27}-10^{28} \sec [25,40,41]$, which should be obviously longer than the cosmic age $\sim 10^{18} \mathrm{sec}$ [42]. Among the theoretical particle physics models of the DM scenarios, some groups have considered a DM particle decaying into two-body and/or multibody daughter particles which produces line and broad spectra of the highenergy neutrinos in order to fit the data $[20,24,26,28,43]$ (see also Refs. [44-46] for alternative models).

However, $\gamma$-ray constraints are crucial for many DM models. There are numbers of DM models that include $\gamma$ rays, charged leptons, quarks and so on as the final states of the decaying DM. Whatever the decay products are, the resulting $\gamma$-ray emission should not violate the observed diffuse $\gamma$-ray flux. For example, charged leptons can easily be converted into secondary $\gamma$-rays at lower energies, e.g., through the inverse Compton scattering off the background photons, as is the case with astrophysical situations. Quarks also emit $\gamma$ rays because they hadronize during their propagation, then, fragment into a lot of pions that immediately decay into a broad spectrum of $\gamma$-rays, charged leptons, and neutrinos. As a result, stringent constraints are placed by the Fermi $\gamma$-ray data $[29,33]$ as well as non-observations of diffuse sub-PeV $\gamma$-rays in air-shower experiments such as KASCADE and CASAMIA [29,47-50]. In particular, Ref. [33] performed thorough analyses on the heavy DM that decays into all final states of standard model particle-antiparticle pairs via flavor-conserving two-body decay. It is found that neutrinophillic DM needs to avoid the $\gamma$-ray constraints, which is especially the case if the medium-energy excess in the IceCube neutrino flux $[4,5,7,10,11]$ is attributed to decaying DM that gives comparable contributions to Galactic and extragalactic components [29,33,35-37].

The required mass of the $\mathrm{DM}$, a few $\mathrm{PeV}$, also has some tension with the standard thermal relic abundance when we assume that the DM to be an elementary particle. The unitarity of the annihilation cross section put an upper limit on the mass of the DM to be about a hundred TeV [51], which is an order of magnitude smaller. In addition, the energy spectrum measured at IceCube has a board component around $\mathcal{O}(10-100) \mathrm{TeV}$ as well as a peak at PeV. This structure does not seem to be easily explained solely by the decay of the DM.

Under these circumstances, we propose a new decaying DM scenario where the DM is very weakly coupled to the standard model particles so that it is almost stable, but is very strongly coupled to other nonstandard-model particles so that it has a finite size (form factor) to make the annihilation cross section larger than the naive unitarity limit and also to make the broad spectrum of neutrinos possible in addition to the line at $\mathrm{PeV}$. In order to make the discussion concrete, we set up an effective particle physics model where the DM particle interact perturbatively to other particles and thus one can evaluate the shape of the neutrino spectrum. In this scenario, DM decays to neutrinos with few $\gamma$-rays in two branches; in one branch, a fermionic DM particle decays into $\mathcal{O}(10)$ particles including a neutrino. A broad spectrum of the produced neutrino is predicted in this branch. In the other branch, the DM undergoes two-body decay into a neutrino and another invisible particle which leads to a line-like feature of the neutrino spectrum. Negligible amount of photons and charged leptons are produced in this model. This property of the DM leads to line and broad spectra of high-energy neutrinos. If the branching fractions into multi-body and two-body are comparable, one could simultaneously explain both of the neutrino flux around the cutoff of a few $\mathrm{PeV}$ and a peak structure below $\mathcal{O}(1) \mathrm{PeV}$ with satisfying the constraint from the $\gamma$-ray observations. For other mechanisms to fit the complex features of the neutrino spectrum, see [52-57] by neutrino absorptions, or [57-59] by neutrino decays.

The outline of this paper is as follows. The particle physics model of DM is introduced in Sec. II A. In Sec. II B, we explain both astrophysical and cosmological effects on high-energy neutrinos. In Sec. III, we show our results. Finally, Sec. V is devoted to summarize and conclude our findings in this paper. 


\section{THE MODEL}

\section{A. Particle physics models}

The data from the IceCube experiment tells us that there is certainly an unexplored source of high-energy neutrinos in the sky. One important fact in this discussion is that the source of the high energy neutrinos should not emit too many $\gamma$-rays in order to be consistent with the observation of the IGRB. This is a nontrivial constraint for both the astrophysical and DM models.

In this section, we consider a model of decaying DM in which the DM mainly decays into neutrinos but not to charged leptons so that the emission of $\gamma$ rays is highly suppressed. Since the neutrino and the charged lepton form a doublet of the $S U(2)$ weak interactions, $\ell$, in the standard model, the DM cannot be a gauge singlet that would not distinguish the neutrinos and charged leptons unless we include the right-handed neutrinos as the final state $[26,28]$. The simplest possibility to achieve the absence of the charged lepton mode is that the DM is a neutral component $N^{0}$ of an $S U(2)$ doublet, $L$,

$$
L=\left(\begin{array}{c}
N^{0} \\
E^{-}
\end{array}\right)
$$

and couples to the lepton doublet, $l$, as

$$
\mathcal{L}_{X}=-m_{\mathrm{DM}} \bar{L} L+(\epsilon \bar{L} \ell X+\text { H.c. }),
$$

in the Lagrangian. Here we introduced the DM sector particles, $L$ and $X$, respectively as a Dirac fermion with hypercharge $-1 / 2$ and a gauge-singlet real scalar field. In the absence of the second term, the lighter of $N^{0}$ and $E^{-}$is stable since there is no decay channel. A dimensionless parameter $\epsilon$ characterizes the coupling between the DM sector particles and the standard model ones, and we assume that is somehow very much suppressed so that $N^{0}$ has survived today as DM. The mass of the DM is assumed to be $m_{\mathrm{DM}}=\mathcal{O}(\mathrm{PeV})$ to explain the PeV neutrinos detected at the IceCube as the primary decay products. The particle $X$ is in general massive, but we ignore its mass compared to the PeV energy scale in the following discussion for simplicity. Since the interactions between $L$ and the Higgs field in the standard model is suppressed by assumption, the mass splitting between $N^{0}$ and $E^{-}$is mainly through the radiative correction, which makes $E^{-}$a little bit heavier than $N^{0}$ by $\mathcal{O}\left(\alpha m_{W} /(4 \pi)\right) \sim$ $300 \mathrm{MeV}$ [60]. We suppressed the flavor indices of $\ell$ for simplicity. The flavor content of the high energy neutrinos can be arranged arbitrarily by the choice of the flavor dependence of the $\epsilon$ parameter.

We further assume that $X$ promptly decays into some other particle $S$ 's in the DM sector through the following interaction terms:

$$
\mathcal{L}_{\text {int }}=\mathcal{L}_{X}+\frac{1}{M^{3 n-3}} X S^{2 n}+\frac{1}{M_{*}^{3 n-1}} \bar{L} \ell S^{2 n}+\text { H.c. }
$$

Here $S$ denotes a massless Weyl spinor field. [The contraction of the spinor and $S U(2)$ indices are implicit.] By assuming a discrete $Z_{2 n}$ symmetry, one can arrange the $S$ particle to be massless and the above operators to be the lowest dimensional ones. The third term is allowed (or induced) in general in the presence of the second term in Eq. (2) and the second term in Eq. (3). We discuss a possible theoretical background behind this model in the Appendix.

Through those interaction terms, the decaying $N^{0}$ simultaneously produces the line $\left(N^{0} \rightarrow \nu+X\right)$ and the broad spectra $\left(N^{0} \rightarrow \nu+2 n S\right)$ of the active neutrinos $\nu$ if $n$ is large enough. The branching fractions into $X$ and $2 n S$ can be comparable in a model with a strongly coupled DM sector. (See Appendix) Although, in a strongly coupled theory, it is possible to add any higher dimensional operators (or equivalently a form factor) to make the shape of the spectrum arbitrary, we will use a standard description of the decay of weakly coupled particles below in order to make discussions concrete and to get a sense of feasibility to fit the broad spectrum of $0.01-1 \mathrm{PeV}$ neutrinos by the decay of DM.

The lifetime of the DM particle is estimated to be

$$
\Gamma_{\text {line }} \sim \frac{\epsilon^{2}}{16 \pi} m_{\mathrm{DM}}
$$

In order to explain the flux of the PeV-energy line, one needs $\epsilon \sim 10^{-29}$.

If one also tries to fit the broad spectrum at $0.01-1 \mathrm{PeV}$, it is required that $2 n S$ is one of the main decay mode. The size of $M_{*}$, in this case, depends on $n$ as follows. By using the third term of (3), the decay rate (or partial decay width) into the broad $\nu$ mode is approximately estimated to be

$$
\Gamma_{\text {broad }} \sim \frac{1}{16 \pi}\left[\frac{1}{(4 \pi)^{2}}\right]^{2 n-1}\left(\frac{m_{\mathrm{DM}}}{M_{*}}\right)^{2(3 n-1)} m_{\mathrm{DM}},
$$

The partial widths $\Gamma_{\text {line }}$ and $\Gamma_{\text {broad }}$ become comparable when

$$
\epsilon \sim\left(\frac{1}{4 \pi}\right)^{2 n-1}\left(\frac{m_{\mathrm{DM}}}{M_{*}}\right)^{3 n-1},
$$

which is the case when $L, X$, and $S$ are strongly coupled as we can see in the Appendix.

Here, we briefly discuss how the correct amount of $N^{0}$ is produced in the early Universe. Since $N^{0}$ interacts with the standard model particles through the electroweak interactions, the DM sector particles are thermally produced as long as the reheating temperature after inflation exceeds $\mathcal{O}(\mathrm{PeV})$. In the standard freeze-out scenario, when the 
temperature drops below $\mathrm{PeV}$, the pair annihilation process of $N^{0}$ into $X$ or $S$ reduces the number density of $N^{0}$. The relic abundance of the $\mathrm{PeV}$ mass particle $N^{0}$, however, is larger than the observed one, provided the annihilation cross section is within the unitarity limit [51] that puts a upper bound on the DM mass to be approximately a hundred $\mathrm{TeV}$. One simple possibility to reconcile the DM abundance is to assume that the annihilation cross section goes beyond the unitarity limit, which means $N^{0}$ is a composite particle with a finite size rather than an elementary particle. The required size is $r \sim 6 \times 10^{-19} \mathrm{~cm}$ for $\sigma v \sim \pi r^{2} \sim 3 \times 10^{-26} \mathrm{~cm}^{3} / \mathrm{s}$. This is interestingly the size expected from the naive dimensional analysis, $r \sim 4 \pi / m_{\mathrm{DM}}$ for $m_{\mathrm{DM}} \sim 0.4 \mathrm{PeV}$. It is somewhat interesting to note that the mass and the spectrum both point to the strongly coupled nature of the DM.

The violation of the unitarity limit does not mean that the naive dimensional analysis overestimates the cross sections. The unitarity is maintained for each partial waves and adding them up provides the consistent estimates [51]. The estimate of the scattering amplitude in the naive dimensional analysis is based on the assumption that the perturbative expansion breaks down; all levels in the perturbative expansion give contributions of the same order of magnitude. This is known to give good estimates in the low-energy hadron physics. The scattering amplitudes $|\mathcal{M}|$ are estimated to be of order $|\mathcal{M}| \sim(4 \pi)^{2}$ in the naive dimensional analysis while the unitarity limit for each partial wave is $|\mathcal{M}| \lesssim \mathcal{O}(4 \pi)$. Therefore, the annihilation cross section of our DM becomes two orders of magnitude higher than that of the naive unitarity limit. For an example of models to go further beyond the unitarity limit, see Ref. [61]. Another possibility is to assume a dilution of DM by a late-time entropy production due to, for example, a decay of a scalar condensation [62]. Yet another possibility would be the scenario with a low reheating temperature. The production of such heavy DM has been shown to be possible in the previous literature (e.g., $[63,64]$ ), and the detailed cosmological scenarios will be discussed in a separate paper.

We also mention the cosmological history of the heavy charged lepton $E^{-}$. It is natural that $E^{-}$has the same abundance as $N^{0}$ in the early Universe since they are the same particle before the electroweak phase transition. The mass difference between $N^{0}$ and $E^{-}$would be expected to be the order of $\Delta m \sim \alpha m_{W} /(4 \pi)$ with the weak boson mass $m_{W}$, which gives $\mathcal{O}(\Delta m) \sim 300 \mathrm{MeV}$. Then the decay width of $E^{-}$is estimated to be $\Gamma_{E^{-}} \sim G_{\mathrm{F}}^{2} \Delta m^{5} \sim\left(10^{-7} \mathrm{sec}\right)^{-1}$. This means that $E^{-}$had disappeared before the beginning of Big Bang nucleosynthesis.

About cosmological histories of $X$ and $S$, it is expected that $X$ had decayed completely into $S$ 's in a short time. The thermalized $S$ around the energy scale of $\mathrm{PeV}$ are diluted by a late-time entropy production including the one after the QCD phase transition. In this case, we predict a dark radiation component by the relic abundance of $S$ as an effective number of neutrino species $N_{\text {eff }}$ to be of the order of 0.1 , which will be tested by future observations, e.g., through precise CMB and $21 \mathrm{~cm}$ line observations [65].

In a more general setup, only a fraction of DM may consist of $N^{0}$ by the ratio of $N^{0}$ to the total DM density, $f_{N^{0}}=\Omega_{N^{0}} / \Omega_{\mathrm{DM}}$ which ranges $f_{N^{0}}=0-1$ with $\Omega_{i}$ the cosmological $\Omega$ parameter of the $i$-particle. Then, a flux of daughter particles produced by the decaying $N^{0}$ is scaled by a factor of $f_{N^{0}}$. In this situation, hereafter we take this notation as read even if it is not stated explicitly.

\section{B. Neutrino spectra}

As mentioned in Sec. II A, we assume the DM particle $N^{0}$ mainly decays into a neutrino $\nu$ and $2 n$ fermion particles $S$ :

$$
N^{0} \rightarrow \nu+2 n S
$$

Hereafter, we use a positive integer $N \equiv 3 n+1$ instead of $n$. In the massless limit of $\nu$ and $S$, the distribution function of the neutrino can be written as

$f(x)=\frac{1}{\Gamma} \frac{d \Gamma}{d x}=4 N(N-1)(N-2) \cdot x^{2}(1-2 x)^{N-3}$

with

$$
x=E / m_{\mathrm{DM}} \quad\left(0 \leq x \leq \frac{1}{2}\right)
$$

where $\Gamma=\tau^{-1}$ is the total decay width of the DM with its lifetime $\tau$. The distribution function $f(x)$ is normalized so that $\int f(x) d x=1$.

We also consider a mode in which the DM particle decays into two particles including a neutrino. In this case, each particle approximately has the energy equal to a half of the DM mass. While the multibody decay of the DM produces the broad spectrum of the neutrino, this two-body decay leads to a line spectrum. The branching ratio of each mode is,

$$
\mathrm{BR}_{i}=\Gamma_{i} / \Gamma=\frac{\Gamma_{i}}{\Gamma_{\text {line }}+\Gamma_{\text {broad }}} i=\text { line or broad. }
$$

The indices $i=$ "line" and "broad" mean the two-body and multibody decay channels of the DM, respectively.

For a given particle physics model, one can calculate neutrino spectra as follows. We consider the late-time decay of the heavy DM, where both extragalactic and Galactic contributions are relevant. The differential flux per energy, area, time, and solid angle, of the extragalactic component is given by (e.g., [41] and references therein) 
$\Phi^{\mathrm{EG}}(E)=\frac{1}{4 \pi H_{0}} \int d z \frac{1}{\sqrt{\Omega_{\Lambda}+(1+z)^{3} \Omega_{m}}} \frac{\bar{\rho}_{\mathrm{DM}}}{m_{\mathrm{DM}} \tau} \frac{d S}{d E^{\prime}}$,

where $E^{\prime}=(1+z) E, d S / d E^{\prime}=f\left(x^{\prime}\right) / m_{\mathrm{DM}}$ is the primary spectrum, and $\bar{\rho}_{\mathrm{DM}}$ is the DM energy density in the Universe. We adopt $H_{0} \equiv 100 \mathrm{~h}=70.2 \mathrm{~km} \mathrm{~s}^{-1} \mathrm{Mpc}^{-1}$, $\Omega_{\mathrm{dm}}=0.229, \Omega_{m}=0.275$ and $\Omega_{\Lambda}=0.725$ [66] but the results are insensitive to small changes in the cosmological paramaters.

The Galactic component is given by

$$
\Phi^{\mathrm{G}}(E ; \psi)=\frac{R_{\mathrm{sc}} \rho_{\mathrm{sc}}}{4 \pi m_{\mathrm{DM}} \tau} \frac{d S}{d E} \mathcal{J}(\psi)
$$

where the $\mathcal{J}$ factor is

$$
\mathcal{J}(\psi)=\frac{1}{R_{\mathrm{sc}} \rho_{\mathrm{sc}}} \int_{0}^{l_{\max }} d l \rho_{\mathrm{DM}}(r)
$$

where $\rho_{\mathrm{DM}}(r)$ is the DM density profile in the Milky Way, and we use the Navarro-Frenk-White (NFW) profile for the calculations [67]. In the case of decaying DM, contrary to DM annihilation, adopting other profiles do not affect our results significantly. The spatial distribution is nearly isotropic but there is a large-scale anisotropy $[29,47,68,69]$. For the purpose of this work, it is enough to use the average $\mathcal{J}$ factor in a cone with half-angle $\psi$ around the Galactic center:

$$
\mathcal{J}_{\Omega}=\frac{2 \pi}{\Omega} \int_{\cos \psi}^{1} d\left(\cos \psi^{\prime}\right) \mathcal{J}\left(\psi^{\prime}\right)
$$

where $\Omega=2 \pi(1-\cos \psi)$ is the solid angle of a field of view. We use $\rho_{\mathrm{sc}}=0.3 \mathrm{GeV} \mathrm{cm}^{-3}, R_{\mathrm{sc}}=8.5 \mathrm{kpc}$, and $\psi=\pi$ throughout this work.

We include those cosmological modification in the current analyses. However, it is remarkable that approximately it has a peak at $x_{\text {peak }}=E_{\text {peak }} / m_{\mathrm{DM}}=2 /(N+1)$ to be

$$
\begin{aligned}
\left.\Phi(E)\right|_{\text {peak }} \approx & 5.7 \times 10^{-8}\left(\frac{A(N)}{0.04}\right)\left(\frac{\tau}{10^{27} \mathrm{~s}}\right)^{-1}\left(\frac{\mathrm{BR}_{i}}{1}\right)\left(\frac{f_{N^{0}}}{1}\right) \\
& \times\left[\frac{1+1.6\left(\mathcal{J}_{\Omega} / 2\right)}{2.6}\right] \mathrm{GeV} \mathrm{cm}^{-2} \mathrm{~s}^{-1} \mathrm{sr}^{-1},
\end{aligned}
$$

with

$$
A(N)=\frac{2^{6} N(N-1)(N-2)}{(N+1)^{4}}\left(\frac{N-3}{N+1}\right)^{N-3} .
$$

\section{RESULTS}

In this paper, by adopting the above setups for the emission mechanisms of high-energy neutrinos, we study the following two scenarios,

1. Pure DM scenario

2. Hybrid scenario with DM and astrophysical contributions

in the next subsections.

We compare our results with the IceCube data in two ways: the deposit energy distribution of high-energy starting event (HESE) neutrinos [3] and the all-flavor neutrino spectrum obtained by the combined likelihood analysis [5]. The HESE data are composed of high-energy showers and some track samples, which are less contaminated by the atmospheric backgrounds and describe neutrinos above $\sim 60 \mathrm{TeV}$. We first use the four-year HESE data in this work, and calculate the deposit-energy distribution following Ref. [54]. This is more appropriate to see whether a line spectrum is compatible with the data or not. We also use the latter for the specific motivation to discuss the origin of the medium-energy flux excess at around $30 \mathrm{TeV}$. Atmospheric neutrinos are more important below $\sim 100 \mathrm{TeV}$, and more sophisticated analyses with a veto have been performed as well as conventional shower analyses $[4,9]$. In this case, we need more dedicated analyses taking into account the veto efficiency, which is beyond the scope of work. For simplicity, we show the neutrino spectrum data obtained by the combined likelihood analysis [5]. This is justified since in our model, only the broad component from multibody decay could account for the $10-100 \mathrm{TeV}$ data.

\section{A. Pure DM contributions}

First, we consider the case that there is no astrophysical contribution to the observed spectrum of the high-energy neutrinos. We try to fit whole part of the spectrum observed by the IceCube by the line and broad components originated from the decaying DM.

\section{Model 1}

We show in Figs. 1 and 2 the neutrino deposited energy and the source spectrum, respectively, with the parameters, the DM mass, $m_{\mathrm{DM}}=4 \mathrm{PeV}$, the number of daughter particles, $N \sim 30(n=10)$, the lifetime $\tau=1.46 \times 10^{27} \mathrm{sec}$, and the branching fraction into the two-body decay, $\mathrm{BR}_{\text {line }}=0.034$. Although we have not performed quantitative analyses that are beyond the scope of this work, we see a reasonable agreement with the observed spectrum in Fig. 1 for a wide range of deposited energies, $10 \mathrm{TeV}-$ $2 \mathrm{PeV}$, that includes both the line and broad spectrum.

On the other hand, there is some amount of deficit in the lower energy bins around $10-100 \mathrm{TeV}$ in Fig. 2 even for a very large number of $N$. In order to explain the neutrino source spectrum including the medium energies, we may need to assume either additional component such as 


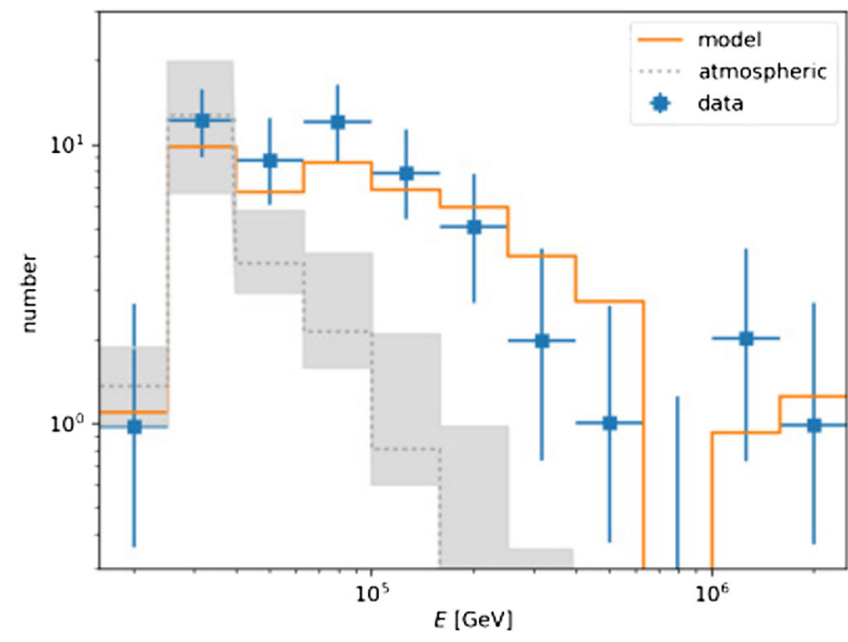

FIG. 1. Neutrino deposited energy histogram predicted by a decaying DM without assuming astrophysical contributions (Model 1). The dots with error bars represent the observed data points. The solid line is the theoretical prediction in the model. We assume that the DM with its mass $m_{\mathrm{DM}}=4 \mathrm{PeV}$ decays into $N \sim 30$ particles. The branching ratio into the two-body decay is taken to be $\mathrm{BR}_{\text {line }}=0.034$. In this model, the lifetime is fitted to be $1.46 \times 10^{27} \mathrm{sec}$. We also show the atmospheric contribution with short-dashed lines with its uncertainty (gray shaded regions).

astrophysical sources or some level of contaminations by the atmospheric muons.

\section{B. DM + astrophysical contributions}

Motivated by the results in Model 1, we consider cases where an additional astrophysical component contributes to the spectrum. In such a two-component scenario [10], we have two possibilities: the astrophysical component for

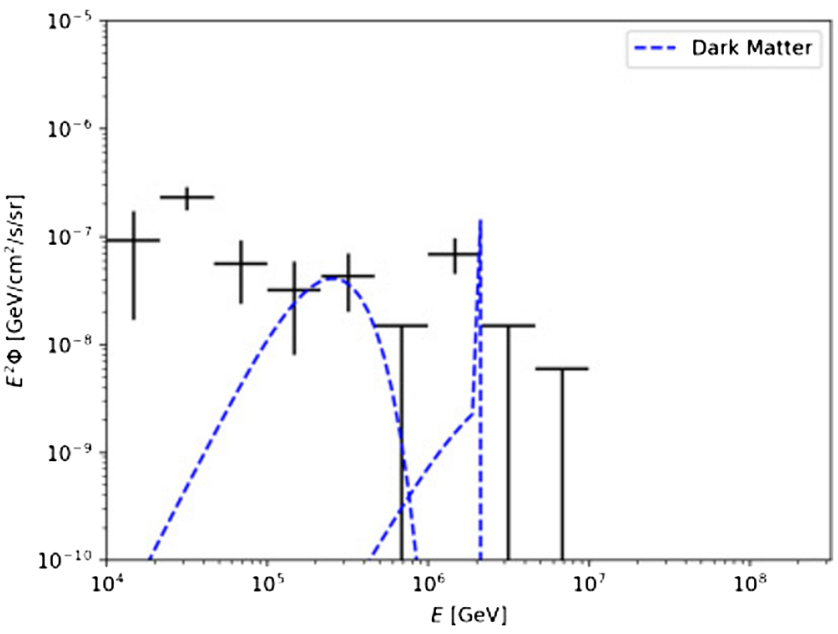

FIG. 2. Neutrino source spectrum in Model 1. Black points with error bars are data. Dashed line represents the model prediction. Model parameters are same in those used in Fig. 1. The IceCube data for the sum of all flavors come from the results of the combined likelihood analysis.
10-100 TeV neutrinos and the DM component for $\gtrsim 100 \mathrm{TeV}$ neutrinos, or vice versa. We first discuss the possibility of filling the deficit in the lower-energy bins by astrophysical sources (Model 2a). Another possibility of having astrophysical component all the way up to $\mathrm{PeV}$ is also examined as Model 2b. Model $2 \mathrm{~b}$ is motivated by a non-trivial success of an astrophysical model. The observed high-energy neutrino flux is remarkably consistent with the Waxman-Bahcall bound with $E_{\nu}^{2} \Phi_{\nu} \sim 3 \times$ $10^{-8} \mathrm{GeV} \mathrm{cm}^{-2} \mathrm{~s}^{-1} \mathrm{sr}^{-1}$ [70]. Astrophysical components are expected to have a power-law spectrum of high-energy neutrinos, and a flat energy spectrum of $E_{\nu}^{2} \Phi_{\nu}$ can explain not only the IceCube data above $0.1 \mathrm{PeV}$ but also the $\gamma$-ray and ultrahigh energy cosmic-ray data [17].

For simplicity, we here adopt a power-law spectrum with an exponential cutoff, $\Phi_{\nu}^{\text {astro }} \propto E^{-s_{\text {astro }}} \exp \left(-E / E_{\text {cut }}\right)$, for an astrophysical component. The spectral index of the astrophysical neutrinos is fixed to $s_{\text {astro }}=2.0$. The cutoff scale is set to $\mathcal{O}(10) \mathrm{TeV}$ (model 2a) and $\mathcal{O}(1) \mathrm{PeV}$ (model 2b), respectively. In the former model, the DM is used for an explanation of the high-energy data, while the mediumenergy data are explained by astrophysical sources (e.g., hidden cosmic-ray accelerators). In the latter model, the astrophysical component explains the data above $0.1 \mathrm{PeV}$, while the medium-energy data are attributed to the DM.

\section{Model 2a: Cutoff of the astrophysical components at $\mathcal{O}(10) \mathrm{TeV}$}

In this model, we consider an astrophysical component for medium-energy neutrinos, which is produced by some hidden accelerators such as choked $\gamma$-ray burst jets and/or cores of active galactic nuclei [17]. We assume that the astrophysical component has a flat energy spectrum of $E_{\nu}^{2} \Phi_{\nu}$ with a cutoff energy at $\mathcal{O}(10) \mathrm{TeV}$. Then, we calculate a total neutrino spectrum by combining the astrophysical component with the broad and line spectra produced by the decaying DM. In Figs. 3 and 4, we show a case that the mass of the DM is $m_{\mathrm{DM}}=4 \mathrm{PeV}$, and the branching ratio into the line is $\mathrm{BR}_{\text {line }}=0.080$. Here we assume that the DM decays into $N \sim 30$ particles with its lifetime $\tau=3.41 \times 10^{27} \mathrm{sec}$.

\section{Model 2b: Cutoff of the astrophysical components at $\mathcal{O}(\mathbf{1}) \mathrm{PeV}$}

The cutoff scale can be taken to be $\sim 2 \mathrm{PeV}$ that is consistent with the nonobservation of the Glashow resonance, and such a spectral suppression was predicted by plausible astrophysical models in which neutrinos are produced in galaxy clusters or starburst galaxies [17]. We show an example of the neutrino spectrum by the combination of such an astrophysical component and the decaying DM contribution. The former (latter) component fits the higher (lower) energy part of the spectrum. In Figs. 5 and 6, we plot the case that the DM particle has its 


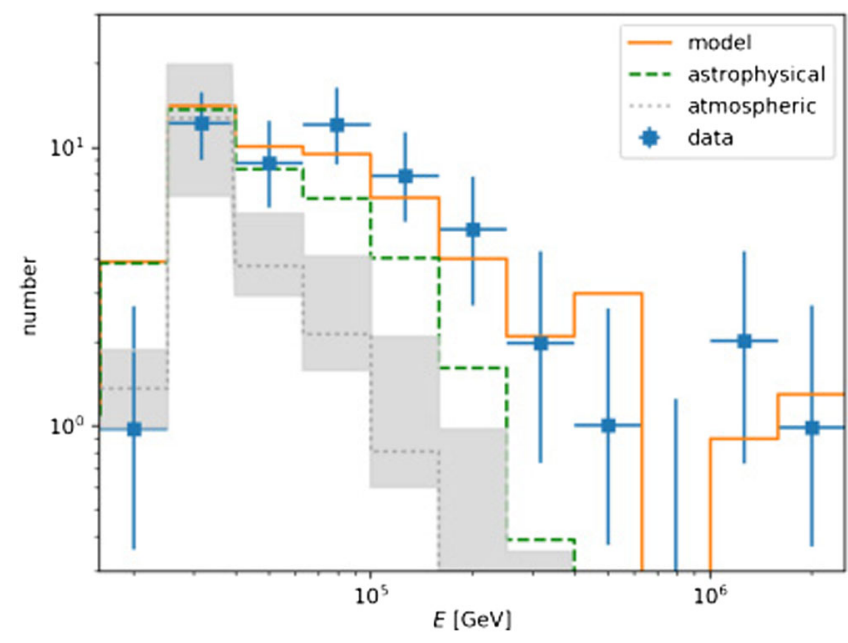

FIG. 3. Deposited energy histogram of the neutrino spectrum combined the astrophysical component with those of the two- and multi-body decaying DM contributions (Model 2a). The total (astrophysical) contribution is represented in the solid (longdashed) line. The short-dashed and shaded region corresponds to the atmospheric contributions and its uncertainty, which is same as those in Fig. 1. In this case, the DM with its mass $m_{\mathrm{DM}}=4 \mathrm{PeV}$ also decays into $N \sim 30$ particles. The branching ratio into the line spectrum and the lifetime is assumed to be $\mathrm{BR}_{\text {line }}=0.080$, and $\tau=3.41 \times 10^{27} \mathrm{sec}$, respectively.

mass $m_{\mathrm{DM}}=600 \mathrm{TeV}$ and decays into $N \sim 30$ particles. Then the lifetime is fitted to be $\tau \sim 5.56=10^{26} \mathrm{sec}$. Here we assume that a line spectrum originated from the twobody decay is negligible.

\section{NOTE ON IGRB}

The multi-body decaying DM model considered in this work is not constrained by the present data of the IGRB

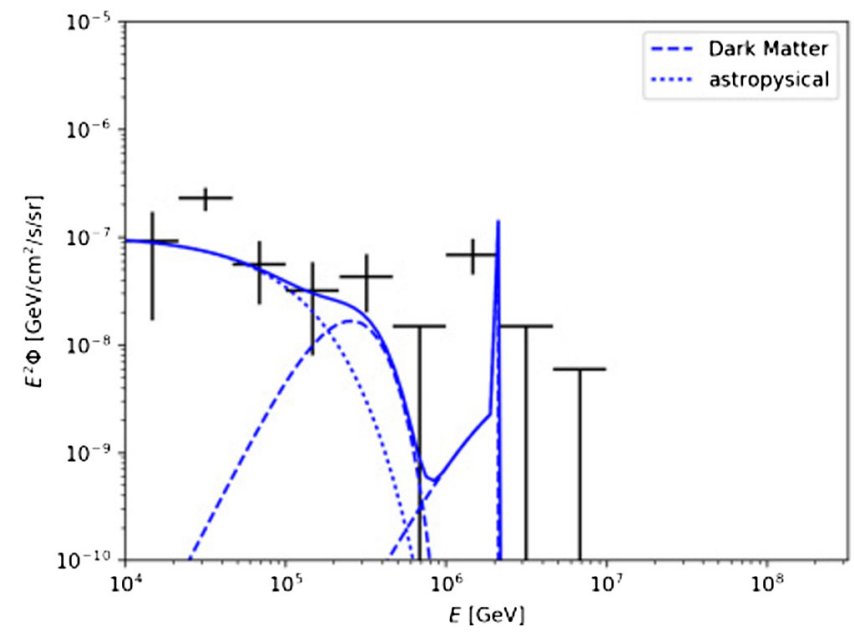

FIG. 4. Neutrino source spectrum in Model 2a corresponds to the Fig. 3. The dashed (dotted) line corresponds to the DM (astrophysical) contributions. The solid line represents the sum of those components.

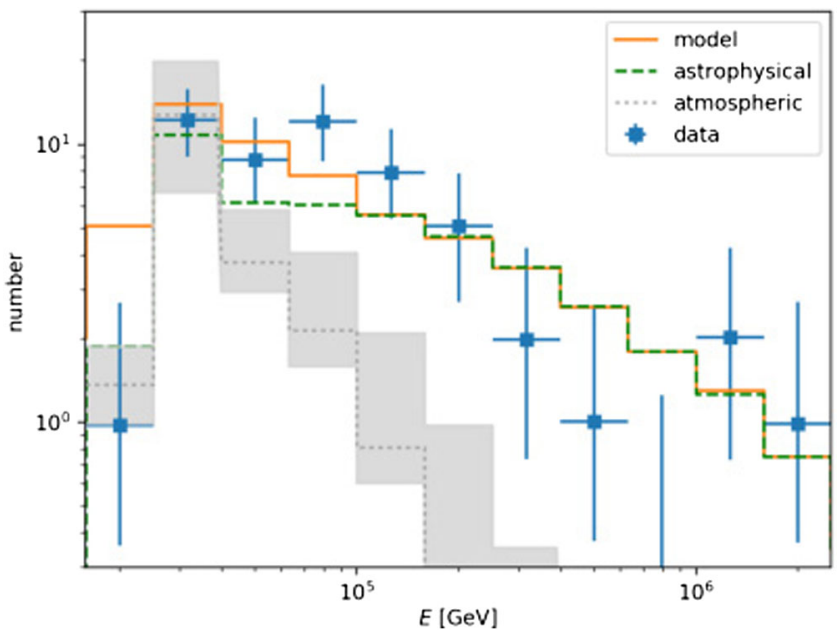

FIG. 5. Deposited energy histogram of the neutrino by both the decaying DM and the astrophysical components (Model 2b). Here we assume that the DM with its mass of $m_{\mathrm{DM}}=600 \mathrm{TeV}$ decays into the $N \sim 30$ particles. Contribution from the two-body decay mode is negligible. In this case, the lifetime of the DM particle is fitted to be $\tau=5.56 \times 10^{26} \mathrm{~s}$. The normalization of the astrophysical component is same as those of [14] in order.

reported by the Fermi satellite, because little $\gamma$-rays are emitted. Electromagnetic emission is expected due to the electroweak bremsstrahlung emission accompanied by the neutrino emissions. We have estimated the contributions to the IGRB in an analytical way. The details of our estimates are as follows, and we show that the contributions to the IGRB is about $1 \%-10 \%$.

We have proposed a DM model which decays to produce neutrinos in two- and multi- particle final states. In each branch of the decay mode, the first next order diagrams, which correspond to the electroweak bremsstrahlung, of the electroweak corrections are

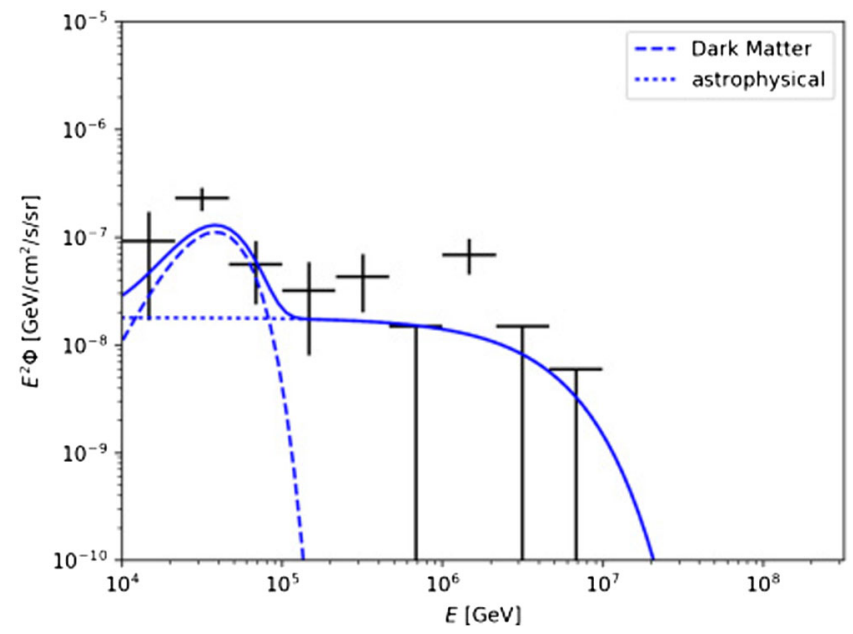

FIG. 6. Source spectrum of the neutrino in Model 2b derived with the same parameters assumed in those of Fig. 5. Lines are the same as those in Fig. 4. 


$$
\begin{aligned}
\nu & \rightarrow \nu+Z \\
& \rightarrow \nu+q \bar{q}
\end{aligned}
$$

and

$$
\begin{aligned}
\nu & \rightarrow W+\text { charged lepton }(e, \mu, \tau) \\
& \rightarrow q \bar{q}+\text { charged lepton }(e, \mu, \tau) .
\end{aligned}
$$

The amplitudes of these corrections are almost same. We take a conservative value as the weak coupling $g \sim 0.65$, then the amplitudes are apploximately

$$
\frac{\alpha_{W}}{\pi} \times \log \left(\frac{1 \mathrm{PeV}}{M_{W}}\right) \sim \mathcal{O}(0.1)
$$

with $\alpha_{W}=g^{2} /(4 \pi)$.

In terms of the flux contributions to the IGRB, inverse Compton emissions from those charged leptons flipped from active neutrinos produced in the electroweak corrections are the most relevant ones. Since the photon spectrum have broad distributions corresponding to the energy distributions of the lepton spectra, the photon flux reduces to a few tens of percent if we look it in each energy bin. Then, we estimate that the contributions to the IGRB flux is at most $10 \%$. Note that this value is derived in an analytical way. Charged leptons are also produced in the decay of the $W / Z$ bosons in the electroweak corrections. In this case, lepton spectra also have broad distributions because they are produced by the decaying mesons which have broad specturm. The reason that those mesons already have broad distributions is they are produced after the hadronizations of $q \bar{q}$ emissions due to the decays of $W$ and/or $Z$. Then, the contributions of the inverse Compton emissions from those leptons to the IGRB is about a few $\%$.

By exactly setting up branching ratios to each modes we can quantitatively estimate the generated $\gamma$-ray flux. In this case, however, the total amount of the $\gamma$-rays are already regulated by the branching ratios in those of analytical estimates we have shown above. For a simplified case of $\mathrm{DM} \rightarrow \nu \nu$, which gives us the most conservative estimate maximising the photon flux at a certain energy bin, Ref. [33] numerically calculated the $\gamma$-ray emissions and showed that the contributions to the IGRB is negligible.

For contributions of the electroweak corrections to the IGRB, those $\gamma$-rays from galactic interactions are dominant. We can neglect the extragalactic contributions at $\mathrm{TeV}-\mathrm{PeV}$ range since the infrared background emissions absorb the $\gamma$-rays higher than $\mathcal{O}(1) \mathrm{TeV}$. Therefore our scenario has not been excluded by the current $\gamma$-ray observations. On the other hand, we may be able to detect these contributions to the IGRB by LHAASO and other future missions. This is beyond the scope of this paper and should be a future work.

\section{DISCUSSION AND CONCLUSION}

We have built models of the multibody decaying DM, in which decay products consist of a neutrino and $\mathcal{O}(30)$ dark fermions. Those neutrinos produced in the decay of the $\mathrm{DM}$ of $m_{\mathrm{DM}} \sim \mathcal{O}(1) \mathrm{PeV}$ can contribute to the TeV-PeV neutrino spectrum. Such a model provides an example of a DM interpretation about high-energy neutrino observations without suffering from the isotropic $\gamma$-ray constraint.

In our setup, the $N^{0}$, which can be a DM candidate, couples to the neutral component of the lepton doublet $l$ in the standard model. Then, $N^{0}$ decays in two modes: (1) one neutrino and $2 n$ fermions $(S)$, and (2) one line neutrino and a scalar $X$ where $X$ decays into $2 n S$. In the first (second) mode, a broad (line) spectrum of neutrino is produced. The observed spectrum should be the sum of the neutrinos produced in these two decay modes.

We can fit the deposited energy histogram of the $\mathrm{TeV}-\mathrm{PeV}$ neutrinos if the mass of $N^{0}$ is $m_{\mathrm{DM}}=4 \mathrm{PeV}$, and $N^{0}$ decays into $N=3 n+1=31$ particles (denoted as Model 1). Then, the lifetime of $N^{0}$ is fitted to be $\tau=$ $1.46 \times 10^{27} \mathrm{~s}$ with its branching ratio into the line spectrum, $\mathrm{BR}_{\text {line }}=0.034$. However, the spectrum produced only by the decaying DM into $\mathcal{O}(30)$ particles may not completely fit the medium-energy feature around $\mathcal{O}(10) \mathrm{TeV}$ in the plot of the neutrino energy spectrum. This indicates that we may need an additional component, such as astrophysical contributions, to explain the whole neutrino spectrum.

Astrophysical neutrinos are assumed to conventionally have a single power-law distribution. Although this is not generally true, the recent IceCube data indicate that the 10-100 TeV flux may not be explained by a single astrophysical component. While various possibilities have been suggested, we have discussed a hybrid model of the astrophysical and the decaying DM contributions. We have studied two cases: the astrophysical components are responsible for (i) the $\sim 30 \mathrm{TeV}$ excess (Model 2a), and (ii) the PeV excess (Model 2b), respectively. In Model 2a, we have assumed neutrinos from astrophysical "hidden accelerators" to fit the $30 \mathrm{TeV}$ excess. On the other hand, the neutrinos produced by the decaying DM contribute only to the $\mathrm{PeV}$ neutrinos. On the other hand, in Model 2b, we have considered the astrophysical neutrinos which are produced in the inelastic $p p$ scattering by cosmic-ray protons with a flat energy spectrum. The decaying DM with $m_{\mathrm{DM}}=$ $600 \mathrm{TeV}$ produces the excess feature of neutrinos at around $E_{\nu}=30 \mathrm{TeV}$. The lifetime of the DM is fitted to be $\tau \sim$ $10^{27} \mathrm{~s}$ in both cases. Note that the astrophysical $\gamma$-rays produced in those models are consistent with the IGRB observations. Our results may imply that we need even such complex scenarios in order to explain the observation of the $\mathrm{TeV}-\mathrm{PeV}$ neutrino, which shows some tension between the low-energy and high-energy data. 
Testing neutrinophillic DM models is challenging in general. One of the promising ways is to search for highenergy neutrino emission from nearby DM halos [29]. If decaying DM scenarios are correct, we should detect neutrino signals from nearby galaxy clusters and galaxies with future neutrino telescopes such as IceCube-Gen2 [71]. Another test is to look for the spatial distribution. Thanks to the Galactic DM component, a slight excess around the Galactic Center is also expected [68]. Discrimination among various DM models is more difficult, but searching for inverse-Compton $\gamma$-rays by leptons from electroweak bremsstrahlung of the Galactic DM is one of the possibilities. We would need a sensitivity of $\sim 10^{-9} \mathrm{GeV} \mathrm{cm}^{-2} \mathrm{~s}^{-1} \mathrm{sr}^{-1}$, which could be reached by LHASSO. Note that dark fermions in our model can be regarded as a boosted DM [38,72-76]. However, their coupling to standard model particles are so weak that it is difficult to detect them with direct detection experiments unless additional assumptions are made.

The model also has some cosmological implications. In the early Universe, $S$ can be thermalized. Then, we predict a dark radiation component of the relic abundance of $S$ to be an effective number of neutrino species $N_{\text {eff }} \sim 0.1$, which could be measured by the future $\mathrm{CMB}$ and $21 \mathrm{~cm}$ line observations [65]. The DM interpretations of the highenergy neutrinos discussed in this paper could be tested in the near future.

\section{ACKNOWLEDGMENTS}

This work is partially supported by Japanese Society for the Promotion of Science (JSPS) KAKENHI Grants No. $15 \mathrm{H} 03669$ and No. $15 \mathrm{KK} 0176$ (R. K.), No. 26247042 and No. JP1701131 (K. K.), and Ministry of Educations, Culture, Sports, Science and TechnologyJapan (MEXT) KAKENHI Grants No. 25105011 (R. K.), No. JP15H05889 and No. JP16H0877 (K. K.). The work of K. M. is supported by NSF Grant No. PHY-1620777.

\section{APPENDIX: A POSSIBLE SCENARIO FOR THE PARTICLE PHYSICS MODEL}

In this section, we describe a setup of particle physics models of a strongly interacting massive sector which includes the heavy lepton $S U(2)$ doublet $L$, the massive scalar $X$, and the massless fermion $S$. We assume a presence of a $Z_{2 n}$ symmetry under which $S$ has a unit charge as in Table I. We set the dynamical scale to be $\Lambda \sim \mathrm{PeV}$.

TABLE I. Charge assignments under the $Z_{2 n}$ symmetry.

\begin{tabular}{llll}
\hline \hline & $S$ & $X$ & $L$ \\
\hline$Z_{2 n}$ & 1 & 0 & 0 \\
\hline \hline
\end{tabular}

According to the naive dimensional analysis which makes the classical estimates and the quantum corrections the same order, any mass scales commonly become the same order of magnitude $[\sim \mathcal{O}(\Lambda)]$. Such a system can be described as

$\mathcal{L}=\frac{\Lambda^{4}}{(4 \pi)^{2}} f\left(L / \Lambda^{3 / 2}, X / \Lambda, S / \Lambda^{3 / 2}, \partial / \Lambda\right)+\mathcal{L}_{\mathrm{SM}}$,

where the symbol " $\partial$ " means the operator of derivative, and $\mathcal{L}_{\mathrm{SM}}$ is the Lagrangian only for the particles in the standard model. Here $f$ is a generic function with taking dimensionless arguments.

From the scaling rule in the first term of (A1), we expect the kinetic term of $X$ can be expressed by

$$
\mathcal{L}_{\text {kin }} \sim \frac{\Lambda^{4}}{(4 \pi)^{2}}\left(\frac{\partial X}{\Lambda^{2}}\right)^{2}
$$

Thus, in order to obtain the canonically normalized kinetic term, we see that $X$ should be multiplied by a factor of $4 \pi$,

$$
X \rightarrow 4 \pi X
$$

This scaling rule must be also applied to any fields in this sector such as $L$ or $S$.

When we discuss the interaction term between $L$ and $\ell$, we introduce a dimensionless parameter $\epsilon$ such as

$$
\mathcal{L}_{1} \sim \epsilon \frac{\Lambda^{4}}{(4 \pi)^{2}} \frac{\bar{L}}{\Lambda^{3 / 2}} \frac{\ell}{\Lambda^{3 / 2}} h\left(X / \Lambda, S / \Lambda^{3 / 2}\right),
$$

with a dimensionless function $h$. Here $\epsilon$ represents the coupling between the DM sector and $\ell$. The interaction term among $L, \ell$, and $X$, are given by

$$
\mathcal{L}_{\text {line }}=\epsilon \frac{\Lambda^{4}}{(4 \pi)^{2}} \frac{\bar{L}}{\Lambda^{3 / 2}} \frac{\ell}{\Lambda^{3 / 2}} \frac{X}{\Lambda} .
$$

By using the scaling rule (A3) for $L$ and $X$, we see that the canonically normalized interaction term is represented by

$$
\mathcal{L}_{\text {line }}=\epsilon \bar{L} \ell X .
$$

In the same manner, the interaction Lagrangian for the mode into a broad spectrum is expressed by

$$
\mathcal{L}_{\text {broad }}=\epsilon \frac{\Lambda^{4}}{(4 \pi)^{2}} \frac{\bar{L}}{\Lambda^{3 / 2}} \frac{\ell}{\Lambda^{3 / 2}}\left(\frac{S}{\Lambda^{3 / 2}}\right)^{2 n} .
$$

Due to the scaling rule (A3), the rescaled one is written as

$$
\mathcal{L}_{\text {broad }}=\epsilon \frac{(4 \pi)^{2 n-1}}{\Lambda^{3 n-1}} \bar{L} \ell S^{2 n} .
$$

The $X$ and $S$ fields are also interacting each other. In total, the Lagrangian with the canonically normalized fields is given by 


$$
\mathcal{L} \sim \Lambda \bar{L} L+\epsilon \frac{(4 \pi)^{2 n-1}}{\Lambda^{3 n-1}} \bar{L} \ell S^{2 n}+\epsilon \bar{L} \ell X+\frac{(4 \pi)^{2 n-1}}{\Lambda^{3 n-3}} X S^{2 n}+\text { H.c. }+\cdots
$$

Compared with Eqs. (2) and (3), we obtain

$$
m_{\mathrm{DM}} \sim \Lambda, \quad \epsilon \sim\left(\frac{1}{4 \pi}\right)^{2 n-1}\left(\frac{\Lambda}{M_{*}}\right)^{3 n-1}, \quad M^{3 n-3} \sim \frac{\Lambda^{3 n-3}}{(4 \pi)^{2 n-1}} .
$$

[1] M. Aartsen et al. (IceCube Collaboration), Phys. Rev. Lett. 111, 021103 (2013).

[2] M. Aartsen et al. (IceCube Collaboration), Science 342, 1242856 (2013).

[3] M. Aartsen et al. (IceCube Collaboration), Phys. Rev. Lett. 113, 101101 (2014).

[4] M. Aartsen et al. (IceCube Collaboration), Phys. Rev. D 91, 022001 (2015).

[5] M. G. Aartsen et al. (IceCube Collaboration), Astrophys. J. 809, 98 (2015).

[6] M. G. Aartsen et al. (IceCube Collaboration), Phys. Rev. Lett. 115, 081102 (2015).

[7] M. G. Aartsen et al. (IceCube), Astrophys. J. 833, 3 (2016).

[8] F. Halzen, Nat. Phys. 13, 232 (2016).

[9] M. G. Aartsen et al. (IceCube Collaboration), in Proceedings, 34th International Cosmic Ray Conference (ICRC 2015) (2015) [arXiv:1510.05223].

[10] C.-Y. Chen, P. S. Bhupal Dev, and A. Soni, Phys. Rev. D 92, 073001 (2015).

[11] A. C. Vincent, S. Palomares-Ruiz, and O. Mena, Phys. Rev. D 94, 023009 (2016).

[12] A. Palladino, M. Spurio, and F. Vissani, J. Cosmol. Astropart. Phys. 12 (2016) 045.

[13] M. Aartsen et al. (IceCube Collaboration), Phys. Rev. Lett. 114, 171102 (2015).

[14] K. Murase, M. Ahlers, and B. C. Lacki, Phys. Rev. D 88, 121301 (2013).

[15] W. Winter, Phys. Rev. D 88, 083007 (2013).

[16] K. Murase, D. Guetta, and M. Ahlers, Phys. Rev. Lett. 116, 071101 (2016).

[17] K. Murase and E. Waxman, Phys. Rev. D 94, 103006 (2016).

[18] K. Fang and K. Murase, arXiv:1704.00015.

[19] M. Ackermann et al. (Fermi LAT Collaboration), Astrophys. J. 799, 86 (2015).

[20] B. Feldstein, A. Kusenko, S. Matsumoto, and T. T. Yanagida, Phys. Rev. D 88, 015004 (2013).

[21] A. Esmaili and P. D. Serpico, J. Cosmol. Astropart. Phys. 11 (2013) 054.

[22] Y. Bai, R. Lu, and J. Salvado, J. High Energy Phys. 01 (2016) 161.

[23] A. Bhattacharya, M. H. Reno, and I. Sarcevic, J. High Energy Phys. 06 (2014) 110.

[24] T. Higaki, R. Kitano, and R. Sato, J. High Energy Phys. 07 (2014) 044.
[25] C. Rott, K. Kohri, and S. C. Park, Phys. Rev. D 92, 023529 (2015).

[26] C. S. Fong, H. Minakata, B. Panes, and R. Z. Funchal, J. High Energy Phys. 02 (2015) 189.

[27] A. Esmaili, S. K. Kang, and P. D. Serpico, J. Cosmol. Astropart. Phys. 12 (2014) 054.

[28] E. Dudas, Y. Mambrini, and K. A. Olive, Phys. Rev. D 91 , 075001 (2015).

[29] K. Murase, R. Laha, S. Ando, and M. Ahlers, Phys. Rev. Lett. 115, 071301 (2015).

[30] M. Re Fiorentin, V. Niro, and N. Fornengo, J. High Energy Phys. 11 (2016) 022.

[31] P. S. B. Dev, D. Kazanas, R. N. Mohapatra, V. L. Teplitz, and Y. Zhang, J. Cosmol. Astropart. Phys. 08 (2016) 034.

[32] P. Di Bari, P. O. Ludl, and S. Palomares-Ruiz, J. Cosmol. Astropart. Phys. 11 (2016) 044.

[33] T. Cohen, K. Murase, N. L. Rodd, B. R. Safdi, and Y. Soreq, Phys. Rev. Lett. 119, 021102 (2017).

[34] D. Borah, A. Dasgupta, U. K. Dey, S. Patra, and G. Tomar, J. High Energy Phys. 09 (2017) 005.

[35] M. Chianese, G. Miele, S. Morisi, and E. Vitagliano, Phys. Lett. B 757, 251 (2016).

[36] M. Chianese, G. Miele, and S. Morisi, J. Cosmol. Astropart. Phys. 01 (2017) 007.

[37] M. Chianese and A. Merle, J. Cosmol. Astropart. Phys. 04 (2017) 017.

[38] A. Bhattacharya, R. Gandhi, A. Gupta, and S. Mukhopadhyay, J. Cosmol. Astropart. Phys. 05 (2017) 002.

[39] P. Ko and Y. Tang, Phys. Lett. B 751, 81 (2015).

[40] A. Esmaili, A. Ibarra, and O. L. Peres, J. Cosmol. Astropart. Phys. 11 (2012) 034.

[41] K. Murase and J. F. Beacom, J. Cosmol. Astropart. Phys. 10 (2012) 043.

[42] S. Palomares-Ruiz, Phys. Lett. B 665, 50 (2008).

[43] S. M. Boucenna, M. Chianese, G. Mangano, G. Miele, S. Morisi, O. Pisanti, and E. Vitagliano, J. Cosmol. Astropart. Phys. 12 (2015) 055.

[44] Y. Ema, R. Jinno, and T. Moroi, Phys. Lett. B 733, 120 (2014).

[45] Y. Ema, R. Jinno, and T. Moroi, J. High Energy Phys. 10 (2014) 150.

[46] Y. Ema and T. Moroi, Phys. Lett. B 762, 353 (2016).

[47] M. Ahlers and K. Murase, Phys. Rev. D 90, 023010 (2014).

[48] O. E. Kalashev and S. V. Troitsky, Pis'ma Zh. Eksp. Teor. Fiz. 100, 865 (2014) [JETP Lett. 100, 761 (2015)].

[49] A. Esmaili and P. D. Serpico, J. Cosmol. Astropart. Phys. 10 (2015) 014. 
[50] O. K. Kalashev and M. Yu. Kuznetsov, Phys. Rev. D 94, 063535 (2016).

[51] K. Griest and M. Kamionkowski, Phys. Rev. Lett. 64, 615 (1990).

[52] K. Ioka and K. Murase, Prog. Theor. Exp. Phys. 2014, 61E01 (2014).

[53] K. C. Y. Ng and J.F. Beacom, Phys. Rev. D 90, 065035 (2014).

[54] K. Blum, A. Hook, and K. Murase, arXiv:1408.3799.

[55] M. Ibe and K. Kaneta, Phys. Rev. D 90, 053011 (2014).

[56] T. Araki, F. Kaneko, Y. Konishi, T. Ota, J. Sato, and T. Shimomura, Phys. Rev. D 91, 037301 (2015).

[57] I. M. Shoemaker and K. Murase, Phys. Rev. D 93, 085004 (2016).

[58] M. Bustamante, J. F. Beacom, and K. Murase, Phys. Rev. D 95, 063013 (2017).

[59] G. Pagliaroli, A. Palladino, F. L. Villante, and F. Vissani, Phys. Rev. D 92, 113008 (2015).

[60] N. Nagata and S. Shirai, J. High Energy Phys. 01 (2015) 029.

[61] K. Harigaya, M. Ibe, K. Kaneta, W. Nakano, and M. Suzuki, J. High Energy Phys. 08 (2016) 151.

[62] R. J. Scherrer and M. S. Turner, Phys. Rev. D 31, 681 (1985).

[63] D. J. H. Chung, E. W. Kolb, and A. Riotto, Phys. Rev. Lett. 81, 4048 (1998).
[64] D. J. H. Chung, E. W. Kolb, and A. Riotto, Phys. Rev. D 59, 023501 (1998).

[65] K. Kohri, Y. Oyama, T. Sekiguchi, and T. Takahashi, J. Cosmol. Astropart. Phys. 10 (2013) 065.

[66] E. Komatsu et al. (WMAP Collaboration), Astrophys. J. Suppl. Ser. 192, 18 (2011).

[67] J. F. Navarro, C. S. Frenk, and S. D. White, Astrophys. J. 490, 493 (1997).

[68] Y. Bai, A. J. Barger, V. Barger, R. Lu, A. D. Peterson, and J. Salvado, Phys. Rev. D 90, 063012 (2014).

[69] P. B. Denton, D. Marfatia, and T. J. Weiler, J. Cosmol. Astropart. Phys. 08 (2017) 033.

[70] E. Waxman and J. N. Bahcall, Phys. Rev. D 59, 023002 (1998).

[71] M. Aartsen et al. (IceCube-Gen2 Collaboration), Proc. Sci., FRAPWS2016 (2017) 004 [arXiv:1412.5106].

[72] K. Agashe, Y. Cui, L. Necib, and J. Thaler, J. Cosmol. Astropart. Phys. 10 (2014) 062.

[73] A. Bhattacharya, R. Gandhi, and A. Gupta, J. Cosmol. Astropart. Phys. 03 (2015) 027.

[74] J. Kopp, J. Liu, and X.-P. Wang, J. High Energy Phys. 04 (2015) 105.

[75] L. Necib, J. Moon, T. Wongjirad, and J. M. Conrad, Phys. Rev. D 95, 075018 (2017).

[76] K. Kong, G. Mohlabeng, and J.-C. Park, Phys. Lett. B 743, 256 (2015). 